\title{
An Adult Case of Heart Failure due to Left Main Coronary Artery Atresia
}

\author{
Moe Horiuchi $^{\text {a }}$, Kuniyasu Harimoto ${ }^{\text {, }}$, Michiyo Yamano ${ }^{\text {a }}$, Tatsuya Kawasakia, b
}

\begin{abstract}
Left main coronary artery (LMCA) atresia is a rare congenital heart disease and can be fatal in pediatric patients. We report an adult case of LMCA atresia, in which heart failure developed without episodes suggesting angina. A 40-year-old man presented with difficulty breathing. Echocardiography revealed diffuse hypokinesis of the left ventricle with an ejection fraction of $22 \%$ in the absence of significant valvular disease. A diagnosis of heart failure was made, and diuretics, enalapril, bisoprolol and warfarin were administered. Coronary angiography demonstrated no trace of the ostium of the LMCA in the sinuses of Valsalva; the middle to distal part of the LMCA was visualized by rich collateral flow from the right coronary artery to the left anterior descending coronary artery and left circumflex coronary artery. No trace of the ostium of the LMCA from the aorta or main pulmonary artery was detected on computed tomography angiography or echocardiography. The patient underwent coronary artery bypass grafting and a final diagnosis of congenital atresia of LMCA was made. The clinical course was uneventful and computed tomography angiography, performed 5 days after surgery, showed a patent bypass graft. This case demonstrates the importance of considering LMCA atresia even in the absence of chest symptoms suggesting angina in patients with heart failure.
\end{abstract}

Keywords: Adult; Atresia; Congenital; Left main coronary artery

\section{Introduction}

Congenital anomalies of the coronary arteries are not uncommon and are non-fatal in most cases such as ectopic origin of the coronary arteries from the aortic sinus of Valsalva or separate ostia of the left coronary arteries $[1,2]$. However, attention should be paid to anomalies related to the left main coronary artery (LMCA) because it plays an essential role in providing

Manuscript submitted February 14, 2021, accepted March 1, 2021

Published online March 24, 2021

aDepartment of Cardiology, Matsushita Memorial Hospital, Osaka, Japan bCorresponding Author: Tatsuya Kawasaki, Department of Cardiology, Matsushita Memorial Hospital, Sotojima 5-55, Moriguchi, Osaka 570-8540, Japan.Email: js-k@wf6.so-net.ne.jp

doi: https://doi.org/10.14740/jmc3681 blood supply to the majority of the left ventricle. We report an adult case of LMCA atresia in which heart failure developed without episodes suggesting angina.

\section{Case Report}

A 40-year-old man was referred to the department of cardiology of our hospital for difficulty breathing. The patient had been in his normal state of health until approximately 2 weeks before presentation, when exertional dyspnea developed and gradually progressed. His previous medical history included hypertension, dyslipidemia and gout. He did not take any medications. The patient did not drink, smoke, or use illicit drugs, and had no known allergies. There was no family history of cardiovascular diseases.

On examination, he was alert and well. His vital signs were normal except for a blood pressure of 130/111 mm Hg. The jugular venous pressure was not high, but gallops were audible at the apex. No pulmonary rales were heard on auscultation and there was no edema in the legs. Electrocardiography demonstrated left ventricular hypertrophy and left atrial dilation. Chest radiography revealed cardiomegaly with a cardiothoracic ratio of $55 \%$ without pleural effusion. The complete blood cell counts were normal. Levels of total bilirubin, aspartate aminotransferase, alanine aminotransferase, creatinine, uric acid and C-reactive protein were $1.7 \mathrm{mg} / \mathrm{dL}, 110$ $\mathrm{U} / \mathrm{L}, 132 \mathrm{U} / \mathrm{L}, 1.67 \mathrm{mg} / \mathrm{dL}, 33 \mathrm{mg} / \mathrm{dL}$ and $0.43 \mathrm{mg} / \mathrm{dL}$, respectively. Although the creatinine kinase level was $540 \mathrm{U} / \mathrm{L}$, the MB enzyme level was $20 \mathrm{U} / \mathrm{L}$ and the high-sensitivity cardiac troponin T level was $0.020 \mathrm{ng} / \mathrm{mL}$ (reference value $<0.100$ ). The level of brain natriuretic peptide was high at $734.9 \mathrm{pg} / \mathrm{mL}$ (reference value $\leq 18.4$ ).

On echocardiography, the left ventricle was diffusely hypokinetic with an ejection fraction of $22 \%$ and enlarged with an end-diastolic diameter of $56 \mathrm{~mm}$, accompanied by mild mitral regurgitation, findings consistent with dilated cardiomyopathy. The transmitral E/A ratio was 2.73 and the E/early diastolic mitral annular velocity ratio of the intraventricular septum was 22.77. The peak velocity of the tricuspid regurgitant was 3.7 $\mathrm{m} / \mathrm{s}$ and the systolic pulmonary artery pressure was estimated to be $63 \mathrm{~mm} \mathrm{Hg}$. The left atrial volume index was high at 48 $\mathrm{mL} / \mathrm{m}^{2}$ (reference: 17 - 32).

A diagnosis of heart failure was made, and diuretics, enalapril, bisoprolol and warfarin were administered. The contractility of the left ventricle improved to an ejection fraction of $52 \%$ and pulmonary hypertension disappeared. Warfarin was 

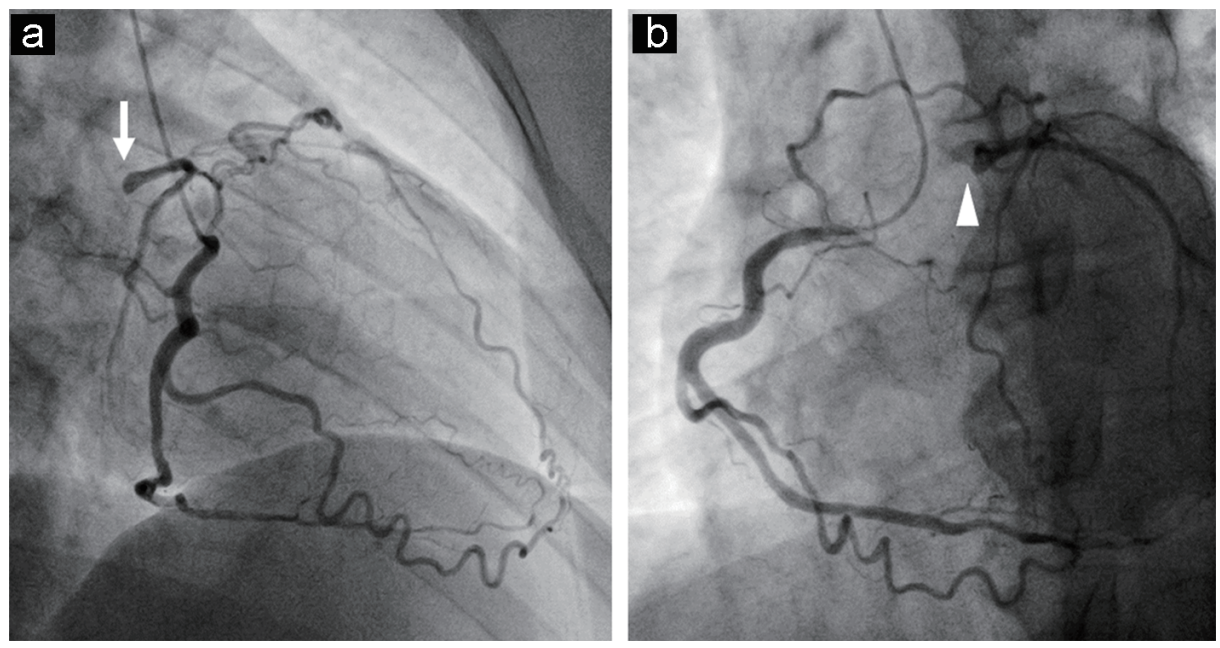

Figure 1. The left anterior oblique $45^{\circ}$ view on coronary angiography shows the rich collateral flow from the right coronary artery to the left anterior descending coronary artery and circumflex artery with the stump of the LMCA ( $a$, arrow), as does the right anterior oblique $35^{\circ}$ view (b, arrowhead).

discontinued. Coronary angiography demonstrated no trace of the ostium of the LMCA in the sinuses of Valsalva; the middle to distal part of the LMCA was visualized by rich collateral flow from the right coronary artery to the left anterior descending coronary artery and left circumflex coronary artery (Fig. 1). No trace of the ostium of the LMCA from the aorta or main pulmonary artery was detected on computed tomography (CT) angiography (Fig. 2) or echocardiography.

Myocardial imaging with thallium (Tl)-201 (111 MBq) and iodine-123 beta-methyl-p-iodophenyl-pentadecanoic acid (I-123 BMIPP) (111 MBq) was performed. A total of $36 \mathrm{im}-$ ages over a $180^{\circ}$ anterior arc were acquired $5 \mathrm{~min}$ and $3 \mathrm{~h}$ after tracer injection. The acquisition lasted 50 beats per projection, was stored in a matrix of $64 \times 64$ pixels and the images were reconstructed using a Hanning filter without attenuation or scatter correction. Bull's eye maps demonstrated mild tracer defect of Tl-201 and I-123 BMIPP in the apex on the initial images, with complete redistribution on the images $3 \mathrm{~h}$ later (Fig. 3 ), suggesting myocardial damage, but not infarction, on the apex in the left ventricle. Of note, the tracer uptake was milder in Tl-201 than in I-123 BMIPP.

The patient underwent coronary artery bypass grafting via an in situ left internal thoracic artery to the mid-left anterior descending artery at another hospital. This surgery was performed through an off-pump approach via left mini-thoracotomy with no signs of myocardial ischemia during the surgery and a final diagnosis of congenital atresia of LMCA was made. The clinical course was uneventful and CT angiography, which was performed at 5 days post-surgery, demonstrated a patent bypass graft and no LMCA with collateral arteries from the right coronary artery (Fig. 4).

\section{Discussion}

The current patient was diagnosed with heart failure at the age of 40 years. Dilated cardiomyopathy was initially suspected as the etiology, but congenital LMCA atresia was later con-
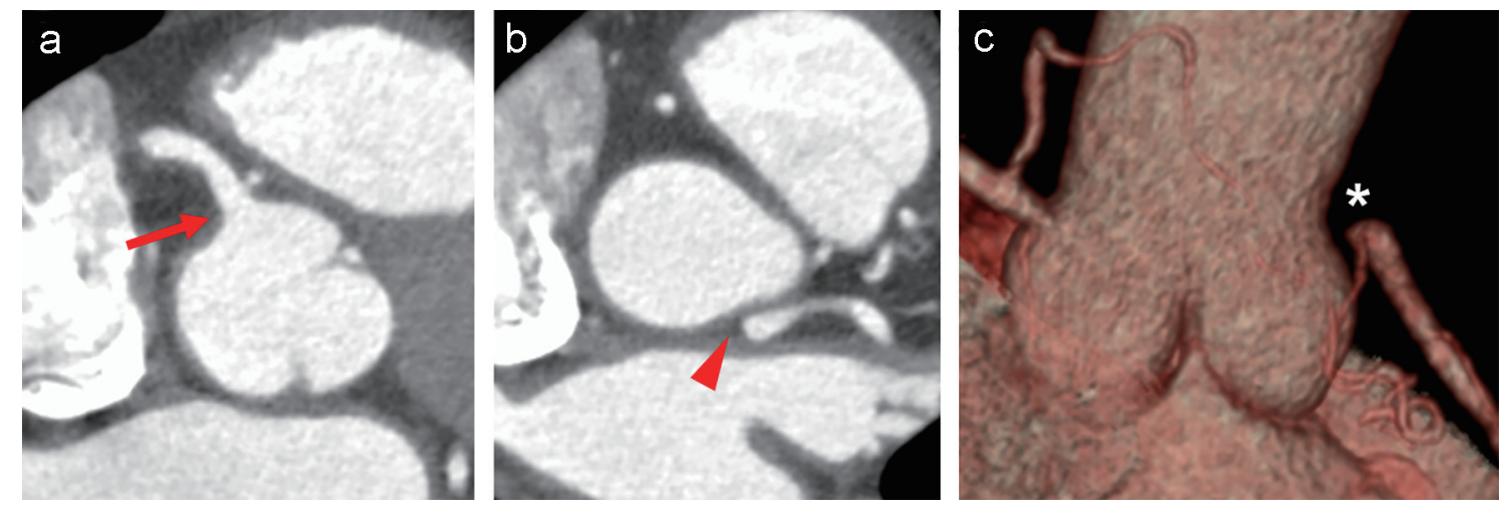

Figure 2. Short axial images of CT obtained after the administration of contrast material show a clear ostium of the right coronary artery ( $a$, arrow), but no connection between the LMCA and the left sinus of Valsalva (b, arrowhead). Note that no trace indicative of the LMCA ostium is detected in the sinus of Valsalva. The absence of the LMCA is shown on the volume rendering image (c, asterisk). CT: computed tomography; LMCA: left main coronary artery. 


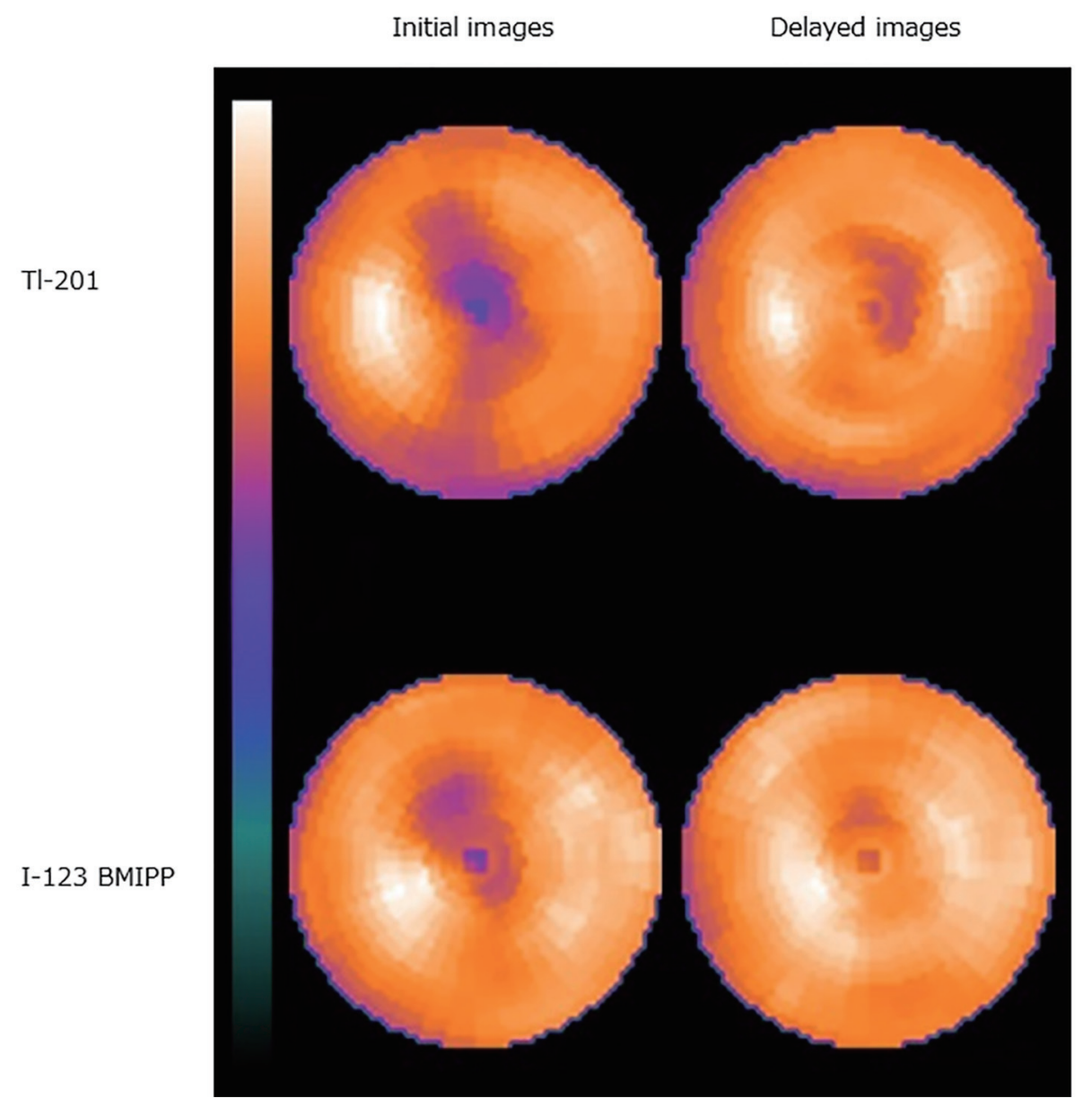

Figure 3. On dual isotope scintigraphy, the Tl-201 bull's-eye map shows reduced tracer uptake in the anterior region to the apex on the initial image with fill-in on the delayed image or $3 \mathrm{~h}$ after injection, findings consistent with ischemic but viable myocardium in the region. Similarly, on the I-123 BMIPP bull's-eye map, decreased fatty acid metabolism (i.e., myocardial ischemia) is noted in the same area. Note that reduced tracer uptake on the initial images is milder with I-123 BMIPP than with TI-201.

firmed. This case is notable because the patient was diagnosed in adulthood, there was a lack of chest pain suggestive of myocardial ischemia and scintigraphy findings were abnormal.

Occlusion of the LMCA can be congenital or acquired. Considering the incidence in the general population and his lipid profile, atherosclerosis was initially considered the most likely cause for the LMCA occlusion despite the absence of chest pain. However, no stump of the LMCA ostium in the sinuses of Valsalva was detected on multiple modalities, including echocardiography, CT angiography and invasive angiography. Furthermore, no atherosclerotic lesion was observed in the coronary arteries except for the LMCA. A diagnosis of congenital LMCA atresia was made based on findings obtained during coronary artery bypass grafting.

The incidence of coronary artery anomalies is estimated to be less than $1 \%$ in the general population, although it varies depending on the definition such as including separate ostia of the left anterior descending and circumflex arteries without the LMCA, myocardial bridge, a high take-off of the coronary ostium and a hypoplastic right or circumflex coronary artery [3-5]. Although the exact incidence of LMCA atresia remains unclear, this condition is considered a markedly rare coronary anomaly that differs from a single coronary artery [6]. LMCA atresia has retrograde blood flow via collateral circulation from the right coronary artery, whereas the blood flow is antegrade in patients with a single coronary artery. In a series of more than one hundred thousand coronary angiograms, coronary anomalies were observed in $1.33 \%$, including $0.04 \%$ in a single coronary artery, but no case of LMCA atresia was found [7].

Bland-White-Garland syndrome or ALCAPA syndrome (i.e., anomalous origin of the left coronary artery from the pulmonary artery) should be included in the differential diagnosis for LMCA atresia $[8,9]$. In the current case, no evidence suggesting the presence of the left coronary artery from the pulmonary artery was obtained on echocardiography focused on the pulmonary artery or cardiac CT. However, the right coronary artery may provide extensive collateral flow to the left anterior descending and circumflex arteries, leading to the disappearance of the LMCA originating from the pulmonary artery if patients survive to adulthood $[10,11]$. This possibility was excluded in this case based on the findings during coro- 


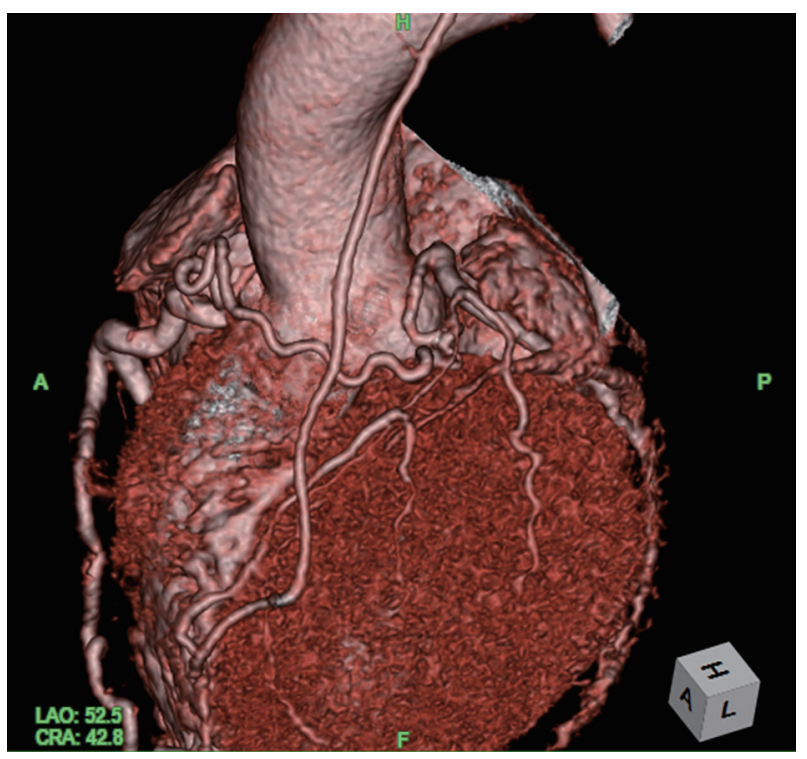

Figure 4. CT angiography after surgery shows that the left anterior descending coronary artery without the LMCA ostium is perfused by the bypassed graft and rich collateral flow from the right coronary artery. Image courtesy of Dr. Satsuki Fukushima. CT: computed tomography; LMCA: left main coronary artery.

nary artery bypass grafting.

LMCA atresia is considered to be associated with a poor prognosis because the blood flow to the left coronary arteries is insufficient for the left ventricle [6]. The majority of patients with LMCA atresia are symptomatic, and sudden cardiac death occurred in $10 \%$ of pediatric patients and $7 \%$ of adult patients [11]. The current patient developed heart failure with reduced ejection fraction, which was managed by medication. Based on the myocardial scintigraphy findings, recovered left ventricular ejection fraction and lack of chest pain, myocardial ischemia was considered minimal with optimal medical treatment. This may be explained by rich collateral flow from the right coronary artery to the left coronary arteries on angiography. Furthermore, this may explain why the current patient survived without symptoms until the age of 40 years. Similarly, there are reports of adults diagnosed with LMCA in their 40 s or later $[12,13]$.

In this case, the uptake of the I-123 BMIPP tracer was greater in the initial images than that of Tl-201. This phenomenon is called paradoxical mismatch and considered to result from an artifact, especially when observed in the inferior wall due to attenuation and infra diaphragmatic scatter [14]. This is also observed in the non-hypertrophied regions of patients with hypertrophic cardiomyopathy [15]. Both conditions are less likely to explain the paradoxical mismatch between I-123 BMIPP and Tl-201 observed in the current case. Of note, the myocardial damage assessed by dual scintigraphy using Tl201 and I-123 BMIPP was less severe in the current patient than in patients with Bland-White-Garland syndrome [16, 17], although the exact mechanisms remain to be elucidated. Further studies are required to evaluate the utility of dual scintigraphy for differentiation.

In conclusion, this case demonstrated the importance of considering LMCA atresia even in the absence of chest symptoms suggesting angina in patients with heart failure.

\section{Acknowledgments}

The authors thank Drs Hiroki Sugihara and Satsuki Fukushima for their comments on scintigraphy interpretation and surgical findings, respectively.

\section{Financial Disclosure}

None to declare.

\section{Conflict of Interest}

None to declare.

\section{Informed Consent}

Not applicable because the manuscript has been sufficiently de-identified to protect the patient.

\section{Author Contributions}

$\mathrm{MH}, \mathrm{KH}, \mathrm{MY}$ and TK contributed to data analysis and study design; $\mathrm{MH}$ and TK wrote the paper; $\mathrm{KH}$ and MY reviewed the paper; all authors gave the final approval.

\section{Data Availability}

The authors declare that data supporting the findings of this study are available within the article.

\section{References}

1. Hauser M. Congenital anomalies of the coronary arteries. Heart. 2005;91(9):1240-1245.

2. Harky A, Noshirwani A, Karadakhy O, Ang J. Comprehensive literature review of anomalies of the coronary arteries. J Card Surg. 2019;34(11):1328-1343.

3. Villa AD, Sammut E, Nair A, Rajani R, Bonamini R, Chiribiri A. Coronary artery anomalies overview: The normal and the abnormal. World J Radiol. 2016;8(6):537555.

4. Angelini P. Coronary artery anomalies: an entity in search of an identity. Circulation. 2007;115(10):1296-1305.

5. Angelini P. Coronary artery anomalies - current clinical issues: definitions, classification, incidence, clinical relevance, and treatment guidelines. Tex Heart Inst J. 2002;29(4):271-278.

6. Musiani A, Cernigliaro C, Sansa M, Maselli D, De Gasp- 
eris C. Left main coronary artery atresia: literature review and therapeutical considerations. Eur J Cardiothorac Surg. 1997;11(3):505-514.

7. Yamanaka O, Hobbs RE. Coronary artery anomalies in 126,595 patients undergoing coronary arteriography. Cathet Cardiovasc Diagn. 1990;21(1):28-40.

8. Pena E, Nguyen ET, Merchant N, Dennie C. ALCAPA syndrome: not just a pediatric disease. Radiographics. 2009;29(2):553-565.

9. Talwar S, Jha AJ, Choudhary SK, Gupta SK, Airan B. Repair of anomalous left coronary artery from pulmonary artery (ALCAPA) beyond infancy. Heart Surg Forum. 2013;16(4):E210-215.

10. Barbetakis N, Efstathiou A, Efstathiou N, Papagiannopoulou P, Soulountsi V, Fessatidis I. A long-term survivor of Bland-White-Garland syndrome with systemic collateral supply: a case report and review of the literature. BMC Surg. 2005;5:23.

11. Alsalehi M, Jeewa A, Wan A, Contreras J, Yoo SJ, Laks JA. A case series of left main coronary artery ostial atresia and a review of the literature. Congenit Heart Dis. 2019;14(6):901-923.

12. Tian M, Wang $\mathrm{X}$, Gao H, Wang L, Hu S. Left main coronary artery atresia with concomitant mitral regurgitation in an adult: A case report. Medicine (Baltimore). 2018;97(41):e12367.

13. Numasawa Y, Motoda H, Yamazaki H, Kuno T, Hashimoto $\mathrm{O}$, Takahashi $\mathrm{T}$. A rare adult case of left main coronary artery atresia. Cardiovasc Interv Ther. 2013;28(3):313317.

14. Ueshima K, Miyakawa T, Taniguchi Y, Nishiyama O, Musha T, Saitoh M, Kamata J, et al. The incidence of discrepant regional myocardial uptake between 201 thallium and 123 I-BMIPP SPECT in patients with coronary heart disease. Int J Cardiovasc Imaging. 2002;18(4):273-278.

15. Ueshima K, Taniguchi Y, Nishiyama O, Saitoh M, Okajima T, Aisaka M, Miyakawa T, et al. Paradoxical regional myocardial uptake between 201Thallium and 123IBMIPP SPECT in patients with cardiomyopathy. Heart Vessels. 2003;18(1):55-56.

16. Yuzawa-Tsukada N, Kashiwagi Y, Fukushima K, Shirasaki K, Oki Y, Kamba T, Kubota T, et al. (123)I-BMIPP/ (201)Tl dual myocardial SPECT proves the efficacy of surgical treatment for an adult with Bland-White-Garland syndrome. J Cardiol Cases. 2020;21(1):39-42.

17. Kanamaru H, Karasawa K, Ichikawa R, Matsumura M, Miyashita M, Taniguchi K, Ayusawa M, et al. Dual myocardial scintigraphy mismatch in an infant with BlandWhite-Garland syndrome. Int J Cardiol. 2009;135(1):e13 . 